\title{
Corela
}

Cognition, représentation, langage

HS-19 | 2016

Le point de vue pris au mot

\section{Langue et valeurs culturelles : six façons d'y voir plus clair}

\section{Bert Peeters}

\section{(2) OpenEdition}

Journals

\section{Édition électronique}

URL : http://journals.openedition.org/corela/4347

DOI : $10.4000 /$ corela.4347

ISSN : 1638-573X

Éditeur

Cercle linguistique du Centre et de l'Ouest - CerLICO

Référence électronique

Bert Peeters, "Langue et valeurs culturelles : six façons d'y voir plus clair », Corela [En ligne], HS-19 | 2016, mis en ligne le 21 septembre 2016, consulté le 01 mai 2019. URL : http:// journals.openedition.org/corela/4347 ; DOI : 10.4000/corela.4347

Ce document a été généré automatiquement le 1 mai 2019.

\section{(c) (i) (2)(2)}

Corela - cognition, représentation, langage est mis à disposition selon les termes de la licence Creative Commons Attribution - Pas d'Utilisation Commerciale - Partage dans les Mêmes Conditions 4.0 International. 


\title{
Langue et valeurs culturelles : six façons d'y voir plus clair
}

\author{
Bert Peeters
}

1 « Vu sous un certain angle, tout dans la langue relève plus ou moins de la culture et tout dans la culture est plus ou moins reflété dans la langue ». Ce n'est pas Pierre-Yves Raccah qui s'exprime ainsi, mais Christine Béal, dans un ouvrage consacré aux interactions quotidiennes en français et en anglais (cf. Béal 2010 : 49-50). Les valeurs culturelles dont il sera question dans cet hommage à notre très cher collègue et ami Pierre-Yves empreignent un grand nombre des ressources linguistiques à la disposition des sujets parlants, tout comme les points de vue au cœur de la SPV (ou Sémantique des Points de Vue) élaborée dans plusieurs écrits de notre confrère (voir par exemple Raccah 2002, 2010) empreignent une proportion importante des mots-de-langue. Elles y sont en quelque sorte ensevelies, comme dans une mine d'or, et il faut savoir les en extraire ${ }^{1}$ puisqu'elles sont susceptibles de fournir des indications précieuses quant aux raisons pour lesquelles la communication interculturelle, en particulier, aboutit parfois à des malentendus.

2 Non que les malentendus soient absents d'autres types de communication. Imaginons une situation, plus ou moins complexe, où deux ou plusieurs personnes se parlent, s'interrogent, se contredisent, échangent des idées, essaient de se convaincre, et ainsi de suite. On dit de ces sujets parlants, ou interlocuteurs, qu'ils s'engagent dans une interaction verbale où, à tour de rôle, ils assument la fonction de locuteur, quand ils prennent la parole, et celle d'interprétant, quand ils la cèdent - à moins qu'ils ne parlent en même temps, auquel cas leurs tours de parole se recoupent, ce qui entre francophones n'est pas rare (voir ci-dessous). Le terme interprétant, proposé en 1992 par Bernard Pottier, paraît préférable à certains autres termes qui ont été utilisés dans la littérature. Auditeur et récepteur sont fréquents, mais risquent de créer l'impression que le message est tout simplement « reçu », sans aucun effort de celui qui le « reçoit ». Or, cela n'est pas le cas : au moment même où Pottier publiait sa Sémantique générale (Pottier 1992), Raccah (1992 : 70) évoquait «l'idée - déjà ancienne - selon laquelle les énoncés ne sont pas des récipients qui "contiendraient" du sens, comme une bouteille contiendrait du vin, mais 
des formes qui provoquent la construction d'un sens (et non pas une re-construction d'un sens pré-existant) chez les destinataires de ces énoncés ». Ce qui n'est pas sans poser des problèmes.

3 En effet, si le devoir du locuteur est de se faire comprendre, de faire passer son message (en règle générale, il ne parle pas tout simplement pour ne rien dire), celui de l'interprétant est de comprendre ce qui vient d'être dit, d'identifier les intentions communicatives du locuteur en vue de réagir d'une façon efficace et avec à-propos. Cette quête de sens, cet acte interprétatif, est d'une complexité qu'on a tendance à sous-estimer dans la mesure où les écueils qui se dressent sur le chemin de l'interprétant sont pour la plupart évités sans que celui-ci s'en rende compte (Peeters 2003a). Mais ils ne le sont pas toujours : parfois ils sont insurmontables ou bien ne sont surmontés qu'au prix d'un effort considérable.

4 À cela s'ajoute que, si l'interaction met en présence des interlocuteurs qui n'appartiennent pas à la même langue-culture, les éventuels malentendus ou échecs communicatifs qui surgissent peuvent être occasionnés par des différences culturelles ignorées (Peeters 2003b, 2009, 2013a). Dans une situation de ce genre, surtout si elle est susceptible de se répéter, il sera utile que les interlocuteurs se familiarisent avec les valeurs culturelles les plus importantes dans la langue-culture de l'autre - ou qu'au moins l'un d'eux fasse un effort allant dans ce sens. Mais comment s'y prendra-t-on? Comment se familiariser avec quelque chose d'aussi intangible qu'un ensemble de valeurs?

\section{Stratégies d'apprentissage de valeurs « étrangères »}

5 Est-ce qu'il y a des stratégies d'apprentissage qui facilitent l'acquisition de valeurs culturelles étrangères? Faut-il retourner à l'école, suivre des cours de langue ? Le résultat sera souvent décevant. Même au bout d'une longue période d'apprentissage en situation scolaire, on risque de n'avoir des valeurs d'une culture étrangère qu'une connaissance au mieux superficielle. La plupart des enseignants n'y consacrent guère de temps et on ne trouve que très peu d'indications pertinentes dans la majorité des méthodes et des manuels de langue.

Peut-on faire confiance aux guides culturels que l'on trouve sur les rayons tourisme de la plupart des librairies? Malheureusement, ils ne sont pas toujours fiables. Quel que soit leur objectif, qu'ils cherchent à divertir ou à instruire, à amuser ou à éduquer, ils sont plus souvent que non le produit de pseudo-spécialistes qui perçoivent et cherchent à interpréter la langue-culture sur laquelle ils se penchent en termes de la leur et de celle du public auquel ils s'adressent, tombant victimes d'un ethnocentrisme subreptice et dangereux qui doit être combattu à tout prix. Il y a des guerres qu'il vaut mieux ne pas mener; il y en a d'autres, comme la guerre contre l'ethnocentrisme, dans lesquelles il ne faut jamais faire l'armistice ${ }^{2}$. Au pire, les guides culturels entérinent des stéréotypes qui sont loin de promouvoir une meilleure compréhension et ne vont guère au-delà des observations plus ou moins spontanées, plus ou moins ponctuelles, que l'on trouve sur les lèvres de ceux qui, ayant participé à des interactions entre locuteurs appartenant à des langues-cultures différentes, ont formé des opinions précipitées basées sur «des incidents particuliers» et «des contextes spécifiques» (Béal 2010: 55). Ces opinions, difficiles à déloger par la suite, sont souvent formulées à l'aide de termes péjoratifs qui se retrouvent des deux côtés mais dont la portée est différente. C'est du moins ce qu'a 
constaté Béal (ibid.: 53-54; voir aussi Béal 1993 : 80) lors de ses entretiens avec des locuteurs français et australiens :

Dans le cas des Français et des Australiens, par exemple, on peut noter que les Australiens trouvent les Français 'égocentriques' (parce qu'ils reprennent la parole à la moindre occasion et veulent à tout prix donner leur opinion sur tout), mais que les Français trouvent, eux aussi, que les Australiens "privilégient leur moi ». Dans ce cas, c'est parce qu'ils font passer ce qu'ils sont en train de faire (personnel ou professionnel) avant toute autre tâche qui attend (...). Les Australiens trouvent également certains Français "hypocrites ", parce que, d'après eux, leurs efforts pour être 'polis' s'évaporent dès qu'ils sont sous tension, preuve que leur politesse n'était pas sincère! Mais les Français trouvent aussi les Australiens «hypocrites » : c'est «leur manie de vous ménager» qui les gêne. En réalité, les Australiens utilisent plus de précautions oratoires et n'expriment pas volontiers une opinion contradictoire, alors que les Français - qui disent ce qu'ils pensent au risque de blesser - se considèrent comme «sincères ». Enfin, les Australiens disent que les Français sont "conformistes ». Dans ce cas-là, c'est le respect de la hiérarchie qui est en cause (les Australiens pensent que si les employés français n'appellent pas leur patron par son prénom, c'est parce qu'ils «n'osent pas »). Mais les Français trouvent aussi les Australiens "conformistes", cette fois, à cause de ce qu'ils considèrent comme des rituels de politesse "désuets " et «qui ne veulent rien dire".

7 Une meilleure façon d'acquérir des valeurs culturelles non natives semble être de s'immerger dans la langue-culture correspondante. Plus qu'en lisant des guides culturels ou en suivant des cours de langue, c'est en passant par une période d'immersion, de contact quotidien avec une langue-culture moins familière, que l'apprenant pourra se rendre compte des différences qui existent par rapport à la langue-culture qui est la sienne. Encore faut-il qu'il comprenne ce qui se passe autour de lui. Ce n'est pas toujours le cas dès le premier jour ${ }^{3}$. D'aucuns ont l'impression, en arrivant, de ne percevoir que ce que Py (2004 : 126) a joliment appelé un « magma verbal indifférencié » (cf. aussi Py 2007). En outre, tout le monde n'a pas les moyens (financiers et autres) de s'offrir un séjour en immersion complète. Finalement, l'immersion, pour être d'une efficacité totale et donner lieu à l'acquisition de valeurs étrangères, doit aller de pair avec un exercice d'observation, d'assimilation et d'imitation de comportements non natifs: le plus souvent, les locuteurs natifs ne sont pas bien placés pour enseigner ou inculquer leurs valeurs à d'autres qui n'en ont pas l'habitude. La transmission de valeurs par enseignement présuppose qu'on est conscient de ce qu'on cherche à transmettre; or, les valeurs culturelles sont souvent si solidement enracinées qu'une telle conscience, simplement, n'existe pas. L'apprenant se voit alors obligé de développer une compétence particulière qu'on a appelée le savoir-apprendre, c'est-à-dire une disponibilité ou une aptitude cognitive à se familiariser, avec plus ou moins de bonheur, avec des savoirs nouveaux, à s'acculturer, et ce d'une façon indépendante, au-delà de tout apprentissage formel. Savoir apprendre, c'est pouvoir apprendre sans se faire enseigner (Gremmo 1995 / 1996). Une fois de plus, tout le monde n'en est pas capable.

8 Acquérir les valeurs qui sous-tendent une conversation entre locuteurs natifs d'une langue étrangère, en même temps que les sons, les mots et la grammaire de cette langue, voilà qui est facile à dire, mais nettement moins facile à faire. Il en est de même des normes qui sous-tendent les comportements communicatifs. Pour la plupart des apprenants, il y a un point limite au-delà duquel il est très difficile, voire impossible, d'acquérir des normes et des valeurs étrangères. Certes, d'aucuns s'efforceront de s'intégrer d'une façon aussi parfaite que possible dans la société d'accueil. Ayant adopté 
(du moins dans leur propre jugement) les normes et les valeurs d'une langue étrangère, ils ont du mal à reconnaître les normes et les valeurs de leur première langue s'il leur arrive d'utiliser celle-ci plutôt que celle-là. Le 09/05/2000, Marie-Odile Junker, linguiste d'origine française vivant à Carleton (Canada), nous adressa un courriel contenant notamment le passage que voici :

Ce qui est drôle, c'est que je reviens d'un voyage en France où j'ai eu du mal à me réadapter au style conversationnel engagé de certains membres de ma famille. J'en sortais épuisée, toute bizarre... Jusqu'à ce que je me souvienne que c'était comme ça, qu'il n'y avait pas de malice de la part de ces gens. Je suppose que j'ai pas mal absorbé sans m'en rendre compte les scénarios culturels de la culture anglophone... ce qui me porte, pour quelques jours Quand j'arrive en Europe, à juger les Français comme une Anglaise. On est si peu conscient de cet aspect du bilinguisme... Avant cela, j'avais bien sûr fait l'expérience du contraire (réactions des anglophones à mon style de conversation française, même quand je parlais anglais). (Correspondance personnelle)

9 Ceux qui cherchent à s'adapter dans les meilleurs délais à leur nouvel environnement se feront peut-être remarquer par un excès de zèle. Dans un devoir d'étudiant soumis le 06/08/1997, Bruno Cayoun, francophone de France expatrié en culture anglophone (australienne), nous rapporta ce qui suit :

À l'époque, je vivais en Australie depuis une période d'environ trois ans pendant laquelle j'avais essayé de m'intégrer à la culture, entre autres par le moyen du langage. Je m'étais donc longuement entraîné à prononcer des mots très représentatifs de la culture australienne, surtout afin de me sentir en Australie comme chez moi ; d'autant plus que de temps en temps ça faisait rire les amis.

Un jour, mon beau-frère, que je ne rencontrais que rarement, nous rendit visite. Le lendemain matin de son arrivée, je l'approchai en lui disant "G'day mate! ». Il sourit et me dit très amicalement que ça ne m'allait pas d'imiter les gens locaux et qu'il valait mieux dire « Hello » ou « Good morning » avec un accent français. Je me suis dit qu'il était snob, mais que d'autres Australiens, moins francs, auraient pu penser la même chose sans me le dire pendant trois ans. Cette première confrontation, quoique passive, me rendit pendant quelque temps douteux à propos de ma capacité d'intégration. (Copie d'étudiant)

Certains refuseront d'inclure dans le processus d'apprentissage de la langue étrangère une assimilation totale des normes communicatives et des valeurs culturelles. Les normes de la langue maternelle et les valeurs associées font effectivement partie de l'identité de l'individu au point que l'adoption d'autres normes et d'autres valeurs risque d'être perçue comme une perte d'identité. Or, comme l'ont remarqué Vogel et Cormeraie (1996:46), « la crainte de perdre son identité est souvent un facteur de raidissement chez les apprenants, et peut paralyser toute tentative de se "désembourber" de la position ethnocentrique de départ ». Ainsi donc, croyant leurs propres normes et valeurs "supérieures » à celles des natifs, certains apprenants prendront soin de se désidentifier. Ils risquent de s'exposer à des malentendus de toutes sortes, et aux conflits et tensions qui en résultent. D'autres développeront, pour la totalité de leurs interactions verbales, un nouvel ensemble de normes et de valeurs, un mélange dont les éléments constitutifs proviennent aussi bien de leur première que de leur deuxième langue. Ce cas s'observe régulièrement chez ceux qui ont l'occasion de s'exprimer autant dans l'une que dans l'autre.

11 À part les cours de langue traditionnels, les guides culturels et l'immersion, qui ont tous leurs limites, y a-t-il d'autres stratégies permettant d'acquérir des valeurs culturelles étrangères? Il y a lieu de croire qu'il y en a au moins une qui vient compléter celles qui ont été énumérées ci-dessus et qui prépare le terrain pour une immersion future : dans le 
cadre d'une formation en langues étrangères, l'exploitation intelligente de certaines ressources de la langue enseignée, l'analyse sélective de certains comportements communicatifs et l'observation de certains faits de société judicieusement choisis permettront à l'enseignant d'équiper ses étudiants avec des savoirs culturels pertinents.

\section{Postulats de base en communication interculturelle}

12 L'hypothèse qu'il en est ainsi nous est venue à l'esprit à la lecture de Wierzbicka (2003 : 69), qui résume de la façon suivante les postulats de base inspirant un nombre grandissant de recherches en communication interculturelle :
1. In different societies, and different communities, people speak differently
2. These differences in ways of speaking are profound and systematic
3. Different ways of speaking reflect different cultural values, or at least different hierarchies of values
4. Different ways of speaking, different communicative styles can be explained and made sense of, in terms of independently established different cultural values and cultural priorities.

\subsection{Premier postulat}

13 Parler différemment, ce n'est pas simplement parler des langues différentes, avec des sons différents, un lexique différent et une grammaire différente. Dans le premier des postulats wierzbickiens, il est question de différences au niveau des habitudes communicatives, des comportements communicatifs. On n'insistera jamais assez sur l'existence de comportements communicatifs qui diffèrent d'une langue-culture à l'autre ${ }^{4}$ . Or, si les façons de parler d'un groupe s'opposent à celles d'un autre, c'est en dernière analyse parce que les deux groupes respectent - ou plutôt tendent à respecter - des normes communicatives différentes. Goddard s'est exprimé au sujet de celles-ci d'une façon à la fois succincte et claire :

In some parts of the world, for example, it is quite normal for conversations to be loud, full of animation, and bristling with disagreement, while, in others, people prefer to avoid contention, to speak in even, well-considered phrases, and to guard against exposure of their inner selves. In some societies, it is considered very bad to speak when another person is talking, while in others, this is an expected part of a co-conversationalist's work. In some places, silence is felt to be awkward and people rush to fill up every spare second with talk, while in others, silence is welcomed. (Goddard $2000: 81$ ).

14 Les normes communicatives sont relativement stables et peu susceptibles d'être modifiées ou abandonnées sous l'influence de normes incompatibles venant d'ailleurs. On pourrait faire valoir, d'une façon très générale, qu'elles prescrivent certains comportements linguistiques et en proscrivent d'autres, ou bien, puisque le respect de ces normes est par définition une question de degré, qu'elles encouragent certains comportements et en découragent d'autres. Personne ne l'a mieux dit que Goddard (1997 : 199), qui signale que les normes communicatives - il les appelle normes culturelles (cultural norms) - « may be followed by some of the people all of the time, and by all of the people some of the time, but [...] are certainly not followed by all of the people all of the time». Cependant, toute dérogation se fait aux risques et périls du locuteur.

Quand il voit que son interlocuteur s'empêtre, le locuteur de langue française a tendance à « offrir le mot juste» (Béal 1993). Il le fait plus volontiers que le locuteur de langue 
anglaise qui, de peur de s'imposer ou de prendre la parole avant qu'il n'en ait le droit, préfère attendre. C'est, on s'en doute, une généralisation. Il ne faut pas croire que ceux qui s'adressent la parole en anglais attendent toujours que le mot qui manque soit suppléé par le locuteur, fût-ce au prix d'un effort extrême. Il ne faut pas croire non plus que ceux qui s'adressent la parole en français viendront immanquablement à la rescousse les uns des autres. Il s'agit de tendances, de comportements qui correspondent à ce qui se fait d'habitude dans le contexte décrit. Dans l'interaction ci-dessous (Kohonen 2004 : 24), Fm, après avoir marqué à trois reprises qu'il continue à prêter attention à ce qu'on lui dit, fait ce que feraient la plupart des Français; Ff (qui s'empêtrait) exprime sa reconnaissance à l'aide d'un simple oui $(\mathrm{Fm}=$ locuteur de langue française ; $\mathrm{Ff}=$ locutrice de langue française $)^{5}$ :

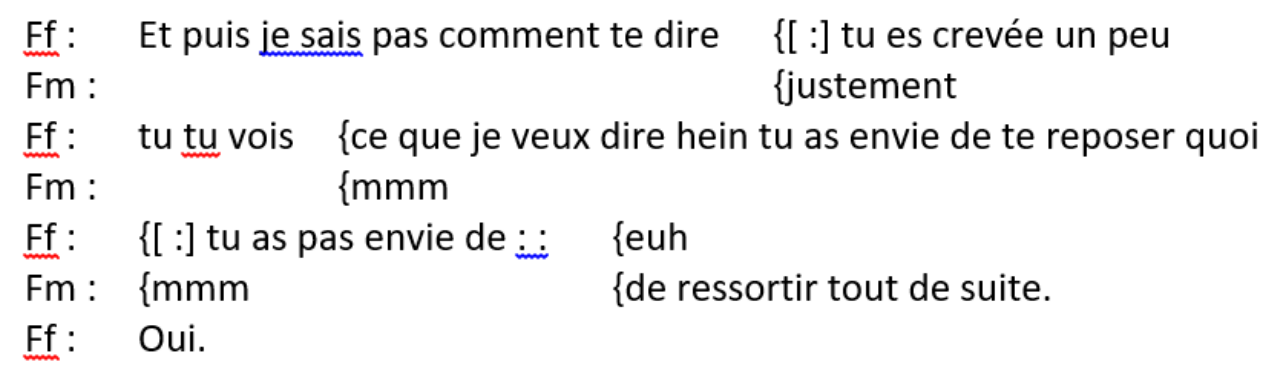

16 Ff aurait pu réagir de plusieurs autres façons, par exemple en disant Voilà, ou encore C'est $c ̧ a$. Des tournures plus longues et plus soutenues (du genre Vous l'avez dit ou bien Vous avez lu ma pensée) sont également possibles (cf. Béal 1993 : 96).

17 Les Français sont en outre enclins à «terminer la phrase de l'autre ». Ce phénomène conversationnel, qui dans la linguistique française a reçu des noms aussi différents que complétion du tour de parole (André-Larochebouvy 1984), achèvement interactif du discours (Gülich 1986) et coénonciation (Jeanneret 1999), transforme un énoncé qui aurait pu être le produit d'un seul locuteur en un produit de locuteur collectif (Loufrani 1981, 1985). Voici un exemple où la socialiste Ségolène Royal, lors d'une interview télévisée, se voit interrompre par son interlocutrice, Françoise Laborde, journaliste ${ }^{6}$ :

$\mathrm{SR}$ : Vous savez, j'ai vu des enseignants - en classe de $4^{\mathrm{e}} !-$ me donner les noms des enfants qui finiraient en prison, à partir de la classe de $4^{\mathrm{e}}$ si rien n'était fait. Est-ce que les socialistes peuvent accepter..

$\mathrm{FL}:$ La faillite du système.

SR : ...que des destins d'enfants se nouent dès la classe de $4^{\mathrm{e}}$ et qu'on sache pertinemment que ces enfants vont finir délinquants? Non! Je pense qu'être socialiste c'est refuser cette fatalité.

Plus que l'offre du mot juste quand un locuteur s'empêtre, l'habitude de terminer les propos d'autrui est susceptible de créer des tensions (ou du moins un certain malaise), surtout si le premier locuteur a l'impression qu'on lui retire la parole alors que le deuxième fait simplement preuve d'intérêt dans ce qu'on lui dit et croit avoir «lu » la pensée de son interlocuteur. Béal $(1993: 89,2010: 169)$ fournit un exemple d'où ressort clairement le malaise du locuteur australien (A), malaise que manifeste non seulement la répétition à l'identique de ce que l'interlocuteur français $(\mathrm{F})$ vient de dire, mais aussi la pause qui suit et la répétition de l'énoncé de départ ${ }^{7}$. 


\section{A : So we have it ready ffor \\ $\mathrm{F}$ : $\quad$ to be corrected \\ A : To be corrected $\quad\{[:]$ so we have it ready for tomorrow afternoon okay \\ $\mathrm{F}$ : $\quad$ yyeah}

19 On a souvent observé que des locuteurs natifs de langue anglaise ont tendance à attendre poliment la fin d'un tour de parole, quitte, s'il le faut, à garder le silence pendant plusieurs minutes. Si ce comportement est adopté dans des interactions verbales en français, avec des locuteurs natifs, ceux-ci ne tarderont pas à se dire qu'il y a quelque chose qui ne va pas. C'est qu'en France le silence est souvent considéré comme gênant. Il peut être symptomatique d'un manque d'intérêt, d'une hésitation à prendre position (Peeters 2000). Peut-être le locuteur français se dira-t-il que son interlocuteur réprouve sa fougue communicative. Le locuteur de langue anglaise, quant à lui, tendra à accuser le francophone d'un manque de politesse, puisqu'il saisit la moindre occasion de prendre la parole et ne saurait attendre son tour.

\subsection{Deuxième postulat}

Pour celui qui s'immerge dans une langue-culture étrangère, le premier des postulats wierzbickiens en matière de communication interculturelle, à savoir "In different societies, and different communities, people speak differently ", devient très vite une réalité incontournable et cesse dès lors d'être un simple postulat. Il n'en est pas nécessairement ainsi dans le cas du deuxième postulat, qui porte sur la nature des divergences: "These differences in ways of speaking are profound and systematic". Postuler une divergence qui est profonde, c'est en souligner l'envergure. Il n'est pas à exclure que les comportements communicatifs observés dans deux langues-cultures apparentées ou non soient relativement proches les uns des autres; cependant, en élargissant l'échantillon des langues-cultures prises en considération, on constatera que les différences ne manqueront pas de devenir de plus en plus manifestes, et on ne tardera guère à trouver des comportements qui sont diamétralement opposés. En fait, il y en a même dans des langues qui ne sont ni typologiquement ni génétiquement très différentes, telles que le français et l'anglais. Prenons le cas du touriste qui se voit offrir quelque chose à boire. S'il est de langue française, et que lors d'un séjour dans un pays anglophone on lui apporte une deuxième tasse de café dont il ne veut pas (Vogel, Cormeraie 1996: 45), c'est qu'il a dit thank you, sans se rendre compte qu'en anglais un remerciement qui suit une offre implique normalement une acceptation plutôt qu'un refus : il voulait dire merci, signifiant 'non merci', mais a utilisé une formule qui, dans le contexte donné, veut dire 'oui, bien sûr' ou 'oui, volontiers'. S'il est de langue anglaise, et qu'il attende en vain un petit cognac qu'on lui a proposé et qu'il ne saurait refuser (Riley 2007 : 193), c'est qu'il a dit merci, sans se rendre compte qu'en français un remerciement qui suit une offre implique normalement un refus plutôt qu'une acceptation : il voulait dire thank you, signifiant 'yes, please', mais a utilisé une formule qui, dans le contexte donné, veut dire 'non, merci'.

21 Est-ce que les différences que l'on constate sont systématiques? Peut-être que dans certains cas, faute de preuves, le doute est permis. Cependant, à ceux qui sont enclins à dire qu'il existe des différences qui sont profondes mais qui manquent de systématicité, on fera remarquer que les langues du monde sont loin de nous avoir livré tous leurs secrets. Il est fort bien possible qu'il y ait une systématicité qui n'a pas encore été 
entièrement dévoilée. En fait, il parait préférable de faire valoir qu'il y a systématicité et de postuler que cette systématicité est grande, plutôt que de décréter - en attendant le jour où quelqu'un sera assez habile pour y voir clair - que c'est l'arbitraire qui règne. La première de ces prises de position invite à la réflexion, la seconde est défaitiste.

\subsection{Troisième postulat}

Le troisième postulat wierzbickien en matière de communication interculturelle attribue la divergence des comportements communicatifs à une divergence plus profonde: "These differences reflect different cultural values, or at least different hierarchies of values ». Reste à savoir ce que cela veut dire... Que faut-il entendre par "valeurs culturelles »? La réponse dépendra évidemment de la définition qu'on donne au terme valeur tout court. Or, observent Bidet et al. (2011: 5), traducteurs français du philosophe américain John Dewey, qui s'est longuement intéressé à la problématique des valeurs, " on peine souvent à trouver une définition précise ». Bréchon (2000 : 9), quant à lui, fait remarquer qu'on a affaire à un «terme galvaudé, dont les usages sont multiples». Il est question de valeurs dans à peu près tous les domaines :

On parle de valeurs culturelles et de valeurs morales, mais aussi de valeurs esthétiques et, bien entendu, de valeurs économiques. Or, qu'ont en commun toutes ces valeurs, si ce n'est le seul usage d'un même terme? Il semblerait ainsi que le succès que rencontre la notion de valeur, y compris dans le débat public et politique, où elle est invoquée à tous propos, tient pour bonne part au flou qui l'entoure. Ce qu'est une valeur relèverait, à l'évidence, d'une sorte de sens commun tacite. (Bidet et al. 2011: 5)

Ce n'est pas pour autant une raison de renoncer à une tentative de définition. Celle de Bréchon, qui sera adoptée ici, relève d'une tradition sociologique illustrée notamment dans les travaux de Jean Stoetzel (1910-1987; voir p.ex. Stoetzel 1983), occupant de la première chaire de psychologie sociale à la Sorbonne, qui concevait les valeurs comme des « positivités » ou des « modèles » :

Dans cette perspective théorique, les valeurs sont des idéaux, des préférences qui prédisposent les individus à agir dans un sens déterminé. Elles appartiennent aux orientations profondes qui structurent les représentations et les actions d'un individu. Elles s'apparentent à ce que certains psychosociologues appellent les « attitudes ». Elles ne sont pas directement observables, à la différence des opinions et des comportements. On peut donc les atteindre par inférence à partir de ce que chacun veut bien livrer de lui-même. Les valeurs d'un individu constituent son identité profonde, ce qui le mobilise et le fait vivre. Dans une telle compréhension, les individus ne perdent pas leurs valeurs. Au cours de leur vie, ils peuvent modifier leurs valeurs de référence, ils peuvent y adhérer avec plus ou moins de force mais ils agissent toujours en fonction d'elles. Les valeurs sont donc un élément primordial de la dynamique de construction des individus et de leurs actions. (Bréchon 2000 : 9)

Dans la formule souvent répétée de John Dewey, susmentionné, les «valeurs modèles » représentent «ce à quoi nous tenons » (en anglais, « what we hold dear »; cf. Bidet et al. 2011). Elles s'opposent aux « valeurs par rapport à un modèle »; ceux-ci sont le résultat d'une évaluation (positive ou négative) rapprochant un objet, une personne, un acte, une pensée etc. d'un modèle qui, lui, fait office d' «étalon de positivité » (Hamon 2008: 10). Les « valeurs modèles ", par contre, font elles-mêmes office d' "étalon de positivité »; il n'y a pas de "valeurs modèles négatives "; ce serait une contradiction dans les termes. Les « valeurs modèles » sont des croyances d'ordre général qui déterminent comment nous 
évaluons des comportements réels ou imaginés de façon à rendre tel comportement approprié, désirable, valorisé, et tel autre inapproprié, indésirable, réprouvé.

À la lumière des observations qui précèdent, on peut définir les valeurs culturelles comme des valeurs largement répandues au sein d'une langue-culture, des valeurs qui soustendent les croyances, les convictions, les attitudes, les habitudes communicatives généralement associées à une langue-culture particulière. Elles n'ont pas toutes la même importance - d'où l'idée d'une hiérarchie de valeurs. Par ailleurs, alors que certaines sont documentées depuis longtemps, d'autres sont restées à l'ombre et commencent seulement à être reconnues en tant que telles. Pour la langue-culture française, on peut citer, parmi les premières, des valeurs telles que la prise de position (Peeters 2000) et l'esprit contestataire (Peeters 2013b); et parmi les secondes, des valeurs telles que la débrouillardise (Peeters à paraitre a) et la méfiance (Peeters à paraître b). Comme on l'a fait remarquer plus haut, les valeurs culturelles risquent d'échapper non seulement à ceux qui ne connaissent que de façon approximative la langue-culture qu'elles contribuent à façonner, mais aussi à ceux qui la vivent "de l'intérieur ». Le plus souvent non perçues en tant que telles, les valeurs culturelles sont donc pour l'essentiel des "évidences invisibles» (Carroll 1987), des évidences qui, comme l'observe d'Iribarne (2006: 287), «alimentent l'étonnement que suscite ce que l'on observe ailleurs ». Il est important de faire remarquer que les valeurs culturelles sont aussi peu absolues que les normes communicatives; cependant, ici aussi, toute infraction risque d'attirer l'attention.

\subsection{Quatrième postulat}

Il reste un quatrième et dernier postulat: "Different ways of speaking, different communicative styles, can be explained and made sense of, in terms of independently established different cultural values and cultural priorities ${ }^{8}$. L'adverbe independently apporte une précision cruciale. Les valeurs culturelles invoquées afin de justifier des comportements communicatifs particuliers ne doivent pas être avancées à la légère, sans référence à d'autres données, linguistiques aussi bien que non linguistiques. Proposer une valeur culturelle en s'appuyant exclusivement sur une ou plusieurs occurrences d'un comportement communicatif équivaudrait à laisser la porte grande ouverte à toutes sortes d'affirmations malvenues. Il faut regarder plus loin, adopter une approche abductive et voir dans toute valeur culturelle postulée ni plus ni moins qu'une conjecture ou une hypothèse qui n'a rien de définitif. La paternité de l'abduction en tant que raisonnement scientifique revient au sémioticien américain Charles Sanders Peirce (1839-1914), cité ici d'après Chmelik (2009: 163) :

Le fait surprenant $C$ est observé ;

Mais si A était vrai, $C$ irait de soi.

Partant, il y a des raisons de soupçonner que A est vrai.

L'abduction est un outil permettant de mieux comprendre des «faits surprenants », par exemple des comportements communicatifs étrangers, en vue de venir à bout du « choc culturel ». Celui-ci ne doit pas être automatiquement perçu de façon négative, comme le fait, parmi plusieurs autres auteurs, Béal (2010) (voir ci-dessus); il peut aussi stimuler la curiosité et fait partie intégrante de l'apprentissage. Dans la définition qu'en donne Cohen-Émerique (1999: 304), le choc culturel est « une réaction de dépaysement, plus encore de frustration ou de rejet, de révolte et d'anxiété, ou sur un mode positif un 
étonnement, une fascination: en un mot, c'est une expérience émotionnelle et intellectuelle qui apparaît chez ceux qui se trouvent engagés dans l'approche de l'étranger ».

Un type très courant d'abduction fait appel au verbe devoir dans un exemple tel que Il a mis trois pulls! Il doit être frileux (Dendale \& Coltier 2013: 48). Le fait surprenant (Il a mis trois pulls) conduit le locuteur à formuler une hypothèse (Il est frileux) qui, corroborée, mettrait fin à la surprise. Étant par définition conjecturale, l'abduction demande à être validée : ce n'est qu'à la lumière d'autres faits (ses notes de chauffage élevées, son refus de partir en vacances de ski...) qu'on pourra conclure que la conjecture ou l'hypothèse est justifiée. De façon analogue, seules des investigations supplémentaires permettent de confirmer la réalité d'une valeur culturelle avancée pour rendre compte d'un comportement communicatif inattendu, divergeant de ceux dont on a l'habitude, mais qui ne serait plus inattendu si la valeur culturelle avancée était réelle ; c'est la seule façon de confirmer ce qui, autrement, est condamné à rester hypothétique. Ces investigations supplémentaires, pour être conclusives, doivent reposer sur des données linguistiques et non linguistiques indépendantes, c'est-à-dire non envisagées au moment de l'étape conjecturale.

\section{Catalyseurs de l'apprentissage}

$\mathrm{Au}$ cœur des quatre postulats évoqués par Wierzbicka (2003: 69) et commentés dans ce qui précède figurent, on l'a vu, des différences d'un type bien particulier. En vertu du troisième postulat, ces différences entre langues-cultures, situées au niveau des comportements communicatifs, sont le résultat d'une adhérence à des valeurs - ou des hiérarchies de valeurs - culturelles différentes. Il s'ensuit que, pour accéder à ces valeurs ou à ces hiérarchies de valeurs, on peut s'appuyer sur l'observation de comportements communicatifs inattendus, insolites ou autrement remarquables, soit sur place (c'est-àdire en immersion), soit (puisque l'immersion n'est pas sans ses limites) auparavant, en cours de langue.

Mais pourquoi s'arrêterait-on là? Ne pourrait-on pas envisager qu'à côté des comportements communicatifs il y ait d'autres faits de langue, dont l'observation est elle aussi susceptible de promouvoir l'acquisition de valeurs culturelles étrangères ? Dans la terminologie de l'anthropologue américain Michael Agar $(1994,1996)$, tous ces faits de langue, y compris les comportements communicatifs, seraient alors des " points riches » ( rich points) ${ }^{9}$, au même titre que certains faits de société marquants, dont l'étude peut vraisemblablement aussi porter des fruits. Les langues cesseraient d'être de simples " moyens de communication"; elles seraient en fait, d'un bout à l'autre, "porteuses de valeurs » (González-Hernández 2012 : 109). L'hypothèse parait tout à fait digne d'intérêt : il n'est pas déraisonnable de faire valoir que, dans le dépistage des valeurs propres à une langue-culture particulière, la langue dans son ensemble peut être mise à contribution, à commencer par le lexique et les séquences figées (c'est-à-dire les tournures courantes, les devises, les slogans, les proverbes, etc.). Comme l'a dit avec beaucoup de justesse Mejri (1997 : 312), « s'il y a une partie de la langue où le culturel trouve sa meilleure expression, c'est bel et bien le lexique et tout particulièrement les séquences figées ». Mais il y a aussi les métaphores, les structures syntaxiques productives... et les comportements communicatifs dont il vient d'être question. Toujours est-il qu'il faudra se garder de conclure que la totalité du lexique, des séquences figées, des métaphores, des structures 
syntaxiques productives et des comportements communicatifs permettent d'accéder à des informations pertinentes. Il s'agit d'identifier les éléments qui sont le plus susceptibles de lever le voile sur les valeurs dont la connaissance est essentielle au succès des interactions verbales. Ces éléments-là pourront servir de clés, de catalyseurs facilitant l'acquisition de valeurs culturelles étrangères. Encore faut-il s'en servir de façon judicieuse, c'est-à-dire sans tomber dans le piège des généralisations hâtives, piège déjà signalé plus haut, quand a été mise en évidence la nécessité de preuves indépendantes en vue de confirmer la réalité de valeurs postulées rien qu'à partir de l'observation de comportements communicatifs particuliers.

31 Une fois qu'on a l'impression d'avoir trouvé un élément prometteur, on peut faire appel à diverses autres ressources (banques de données, ouvrages de référence, littérature spécialisée, ouvrages de vulgarisation, etc.) en vue de déterminer ce que ledit élément peut nous apprendre - si effectivement il peut nous apprendre quoi que ce soit. Reste à voir comment on peut isoler, au sein du lexique et parmi les innombrables séquences figées, métaphores, structures syntaxiques et comportements communicatifs, les candidats catalyseurs les plus solides. Rien de plus simple, à première vue; pour qu'un fait de langue puisse servir de catalyseur, il faut d'abord qu'il ait une visibilité suffisamment élevée. Autrement dit, il doit être saillant. Problème résolu ? Loin s'en faut... Ainsi que le constate notamment Inkova (2011:9) dans l'avant-propos d'un ouvrage collectif consacré aux phénomènes de saillance, «entrée depuis peu dans l'usage linguistique, la saillance n'a pas de définition communément acceptée, et le terme est souvent employée de manière pré-théorique ».

La liste des phénomènes sur lesquels les linguistes ont fait porter la notion de saillance est longue. Celle d'Inkova (ibid.) se limite à « la résolution de l'anaphore, la focalisation, la structure informationnelle, la sémantique verbale, la sélection lexicale, la théorie des prototypes, les effets de style » et se termine par un etc. significatif. Dans tous ces cas, ce qui est visé, c'est « l'importance, l'émergence, ou encore la récence d'une entité dans un contexte donné, propriété qui lui permet d'être perçue, repérée plus facilement au milieu d'autres entités» (ibid.). Inkova ne souffle mot de la saillance culturelle d'un fait de langue ; or, pour quelqu'un soucieux d'en savoir plus long sur les valeurs d'une langueculture différente de la sienne, c'est la seule saillance qui compte. Py (2004:121), pour sa part, déclare saillante toute expression («phonème, syllabe, morphème, syntagme, clause, etc.») qui se trouve "projetée au premier plan dans la perception de l'apprenant »; il est l'un des rares linguistes à s'être occupés de saillance dans le cadre de la didactique des langues étrangères. Lui non plus ne parle de saillance culturelle, mais il en donne néanmoins un exemple. Il fait état du contraste entre les attentes suscitées par un contexte social et une conduite verbale effective : un remerciement qui surgit dans une situation où, dans la langue-culture de l'apprenant, il est inapproprié - alors que, dans cette même situation, il est tout à fait conventionnel dans la langue-culture étudiée - peut attirer l'attention et dès lors être saillant. Cet exemple illustre que, pour l'apprenant, un manque de conventionalité contribue (ou peut contribuer) à la saillance, alors qu'il y a lieu de croire que, pour un locuteur natif, c'est la conventionalité plutôt que son absence qui rend un fait de langue plus saillant (cf. Giora 2003).

Ce qui est saillant pour les uns ne l'est donc pas nécessairement pour les autres. À plusieurs reprises, des étudiants ont attiré notre attention sur des candidats catalyseurs dont ils s'étaient aperçus mais qui, pour une raison quelconque, avaient échappé à son attention. De façon plus générale, un apprenant pourra trouver saillants des éléments qui 
manquent de saillance du point de vue d'un locuteur natif ou pseudo-natif. En outre, il peut y avoir des divergences d'un apprenant ou d'un locuteur natif (ou pseudo-natif) à l'autre. C'est dire que le concept de saillance est empreint de subjectivité. Il s'agit néanmoins, pour l'un comme pour l'autre, d'une subjectivité greffée sur une base qui est indéniablement objective. Adoptant la perspective de l'apprenant, Py (2004:121) apporte la précision suivante :

La saillance se situe à l'articulation entre objectivité et subjectivité, en ce sens que les conditions de la saillance d'un segment se trouvent d'une part dans certaines propriétés objectives du discours (observables), d'autre part dans la manière dont chaque apprenant perçoit, sélectionne et exploite ces propriétés. Les propriétés objectives favorisent plus ou moins la saillance. Mais c'est ensuite le sujet qui sélectionne les candidats à la saillance et institue un ou plusieurs de ces candidats en expression(s) saillante(s).

34 La sélection des "candidats à la saillance ", qu'il s'agisse de saillance culturelle ou de n'importe quelle autre forme de saillance, repose sur une focalisation plus ou moins spontanée: sans qu'il s'en rende compte, l'apprenant (voire le chercheur) opère une distinction entre « ce qui [lui] parait pertinent et utile » et « ce qui lui échappe et/ou dont il n'attend rien» (ibid.: 117). Curieusement, Py ne fait aucune allusion au caractère scalaire de la saillance : à ses yeux, un « segment » est saillant ou bien il ne l'est pas. Or, la saillance étant une notion éminemment scalaire, il va sans dire qu'il y a des degrés de saillance; pour en revenir à la saillance culturelle, plus un fait de langue est (culturellement) saillant, plus il peut prétendre au statut de candidat catalyseur.

35 «Quels sont les critères qui permettent de déterminer qu'une entité est plus saillante qu'une autre et de "mesurer" sa saillance ? Ces critères d'identification de l'entité la plus saillante ont-ils tous la même importance ou certains facteurs sont-ils plus importants que d'autres?» Les questions que soulève Inkova (2011: 9-10) concernent toutes les formes de saillance, y compris la saillance culturelle. Sans aller jusqu'à établir une hiérarchie de facteurs tributaires de cette dernière, on peut commencer par rappeler que, du point de vue de l'apprenant, la saillance se situe plutôt du côté de ce dont on n'a pas (encore) l'habitude. Du coup se trouvent éliminés la quasi-totalité des critères proposés par Giora (2003), qui, adoptant le point de vue du locuteur natif, définit la saillance en termes de fréquence, de familiarité, de conventionalité et de prototypicalité. De ces quatre critères, seule la fréquence entre sans doute en ligne de compte pour l'apprenant : plus celui-ci rencontre dans son environnement langagier un fait de langue quelconque qui l'étonne ou le laisse perplexe, plus il sera amené à y voir l'effet d'une différence culturelle, ce qui est tout ce qu'il faut pour que ce fait de langue devienne un catalyseur potentiel pour l'assimilation de valeurs culturelles étrangères.

Qu'y a-t-il d'autre? Comment procéder en l'absence de stratégies objectives permettant d'identifier une fois pour toutes les catalyseurs qui vont rendre les plus grands services? Un fait de langue dont on parle - c'est-à-dire dont d'autres ont déjà parlé - est par définition plus saillant qu'un autre passé largement inaperçu. Afin d'identifier des candidats catalyseurs pour l'apprentissage de valeurs culturelles étrangères, on peut donc essayer de déterminer dans quelle mesure ils font l'objet de témoignages, internes aussi bien qu'externes. Les témoignages internes sont fournis par des membres de la communauté où la langue-culture prédomine; les témoignages externes, par contre, proviennent d'observateurs qui, comme l'apprenant avancé, regardent la langue-culture étudiée de l'extérieur. En règle générale, les témoignages externes sont plus faciles à repérer que les témoignages internes; on les trouve avec une certaine régularité dans les 
récits de voyage de touristes et dans les récits d'acculturation de nouveaux résidents. Même des romans ou d'autres récits fictifs fournissent parfois des indices précieux. S'il fréquente d'ores et déjà des locuteurs natifs de la langue-culture étudiée, ou s'il a eu l'occasion de le faire, l'apprenant peut par ailleurs se laisser guider par ses propres impressions (cf. plus haut). Finalement, à côté des témoignages, il y a ce qu'on pourrait appeler l'ubiquité du fait de langue dans la langue-culture. L'ubiquité est une forme de fréquence, mesurée en termes d'occurrences dans les titres de librairie, les titres de presse, les chansons, le cinéma, les proverbes, les devises, les slogans etc.

\section{De l'ethnolinguistique à l'ethnolinguistique appliquée}

À la lumière de ce qui vient d'être dit, il paraît possible d'identifier un nombre de démarches distinctes utilisables dans les cours de langue avancés et susceptibles de contribuer à une meilleure compréhension des valeurs culturelles caractéristiques d'une communauté linguistique quelconque (p.ex. les Français, les Touaregs, les Japonais...). Ces démarches sont par ailleurs susceptibles d'intéresser également l'autodidacte et le chercheur. Elles ont été élaborées à l'intention de quiconque désire élargir la gamme des ressources à sa disposition en vue de découvrir et / ou de mieux comprendre des valeurs étrangères à sa propre langue-culture et relèvent de l'ethnolinguistique ${ }^{10}$.

\subsection{Typologie des démarches}

Pour le moment, six démarches - ou ethnodémarches - distinctes sont envisagées : nous les appellerons ETHNOLEXICOLOGIE, ETHNORHÉTORIQUE, ETHNOPHRASÉOLOGIE, ETHNOSYNTAXE, ETHNOPRAGMATIQUE et ETHNOAXIOLOGIE ${ }^{11}$. Les termes à la base de ces étiquettes paraissent assez rébarbatifs; toujours est-il que deux d'entre eux (ethnosyntaxe et ethnopragmatique) ont d'ores et déjà acquis droit de cité au sein de l'approche MSN (métalangue sémantique naturelle), approche indissolublement liée aux travaux d'Anna Wierzbicka et Cliff Goddard. Le recours à la MSN représente le trait le plus saillant de l'ensemble des « ethnodémarches » distinguées ici. Il s'agit d'un outil descriptif culturellement neutre (autant que cela faire se peut), rigoureux et d'une clarté maximale, permettant, grâce à son lexique et sa grammaire empiriquement validés comme étant universels (c'est-à-dire transposables dans toutes les langues du monde sans aucune déformation sémantique), de rendre compte du sens de mots culturellement plus spécifiques, mais aussi d'autres données et faits linguistiques tels que des métaphores, des tournures courantes, des structures syntaxiques, des comportements communicatifs, etc ${ }^{12}$.

1. Sera appelée ETHNOLEXICOLOGIE (Peeters 2013c) la démarche qui consiste à étudier - en invoquant des faits linguistiques aussi bien que non linguistiques - des mots culturellement saillants ${ }^{13}$, en vue de découvrir si derrière ces mots se cachent des valeurs culturelles caractéristiques de la langue-culture où ils ont été observés. Ce n'est pas toujours le cas, mais dans l'affirmatif il peut s'agir soit de valeurs déjà connues, que la démarche ETHNOLEXICOLOGIQUE permettra de mieux comprendre, soit de valeurs précédemment insoupçonnées, hypothétiques, dont il faudra ensuite confirmer la réalité par d'autres moyens. L'ethnolexicologie telle qu'elle vient d'être définie trouve ses origines dans la SÉmANTiQue (TRANS)CULTURELle de Peeters (2003b) et l'ethnosémantiQue de Peeters (2009, 2010a, 2012). 
2. Sera appelée ETHNORHÉTORIQUE (Peeters à paraître c) la démarche qui consiste à étudier - en invoquant des faits linguistiques aussi bien que non linguistiques - des métaphores et d'autres figures de style culturellement saillantes, en vue de découvrir si derrière elles se cachent des valeurs culturelles caractéristiques de la langue-culture où elles ont été observées. Ce n'est pas toujours le cas, mais dans l'affirmatif, il peut s'agir soit de valeurs déjà connues, que la démarche ETHNORHÉTORIQUE permettra de mieux comprendre, soit de valeurs précédemment insoupçonnées, hypothétiques, dont il faudra ensuite confirmer la réalité par d'autres moyens.

3. Sera appelée ETHNOPHRASÉOLOGIE (Peeters 2014) la démarche qui consiste à étudier - en invoquant des faits linguistiques aussi bien que non linguistiques - des tournures culturellement saillantes, en vue de découvrir si derrière ces tournures se cachent des valeurs culturelles caractéristiques de la langue-culture où elles ont été observées. Ce n'est pas toujours le cas, mais dans l'affirmatif il peut s'agir soit de valeurs déjà connues, que la démarche ETHNOPHRASÉOLOGIQUE permettra de mieux comprendre, soit de valeurs précédemment insoupçonnées, hypothétiques, dont il faudra ensuite confirmer la réalité par d'autres moyens.

4. Sera appelée ETHNOSYNTAXE (Peeters 2010b) la démarche qui consiste à étudier en invoquant des faits linguistiques aussi bien que non linguistiques - des structures syntaxiques productives culturellement saillantes, en vue de découvrir si derrière ces structures se cachent des valeurs culturelles caractéristiques de la langue-culture où ils ont été observés. Ce n'est pas toujours le cas, mais dans l'affirmatif il peut s'agir soit de valeurs déjà connues, que la démarche ETHNOSYNTAXIQUE permettra de mieux comprendre, soit de valeurs précédemment insoupçonnées, hypothétiques, dont il faudra ensuite confirmer la réalité par d'autres moyens.

5. Sera appelée ethnopragmatique (Peeters 2013b) la démarche qui consiste à étudier - en invoquant des faits linguistiques aussi bien que non linguistiques - des comportements communicatifs culturellement saillants, en vue de découvrir si derrière ces comportements se cachent des valeurs culturelles caractéristiques de la langue-culture où ils ont été observés. Ce n'est pas toujours le cas, mais dans l'affirmatif il peut s'agir soit de valeurs déjà connues, que la démarche ETHNOPRAGMATIQUE permettra de mieux comprendre, soit de valeurs précédemment insoupçonnées, hypothétiques, dont il faudra ensuite confirmer la réalité par d'autres moyens. L'ETHNOPRAGMATIQUe telle qu'elle vient d'être définie trouve ses origines dans la PRAgmatique (TRANS)CUlturelle de Peeters (2003b) et présente de nombreuses affinités avec la démarche du même nom dans les travaux de Cliff Goddard (voir par exemple Goddard 2006).

6. Sera appelée ETHNOAXIOLOGIE (Peeters à paraître $\mathrm{a}, \mathrm{b}$ ) la démarche qui consiste à confirmer la réalité de valeurs culturelles soit hypothétiques, soit d'ores et déjà associées à une langue-culture particulière, en repérant des faits linguistiques aussi bien que non linguistiques qui en constituent le reflet. Définie de la sorte, 1 'ETHNOAXIOLOGIE trouve ses origines dans l'AXIologie (TRANS)CUlturelle de Peeters (2003b). La démarche ETHNOAXIOlogique sera le plus souvent précédée de l'une des démarches explicitées ci-dessus, mais rien ne s'oppose à ce qu'elle soit effectuée de façon indépendante.

Le nombre de démarches n'est pas définitivement arrêté. Des recherches ultérieures devront déterminer si la liste doit ou peut être allongée. Il convient par ailleurs de signaler qu'au-delà de l'approche MSN les étiquettes proposées ici ne sont pas forcément comprises de la même façon: ethnosyntaxe et ethnopragmatique sont des termes assez répandus et couramment utilisés ailleurs, que ce soit dans le domaine de la linguistique, ou bien dans des domaines tels que la psychiatrie, la psychologie, l'anthropologie ou l'ethnologie. Il importait donc de fournir des définitions. 


\subsection{Vers une ethnolinguistique appliquée} ensemble cohérent. Dans nos publications en langue anglaise, nous y renvoyons à l'aide des étiquettes ETHNolinguistic PATHWAYS Model (Peeters 2009) et APPLIED ETHNOLINGUistics (Peeters 2013a). Dans nos publications en langue française (Peeters 2010a, 2012), nous avons jusqu'il y a peu adopté l'étiquette ETHNOLINGUISTiQue, à l'instar d'autres chercheurs (Riley 2007 ; Bartmiński 2009 ; Underhill 2012) qui, au cours de ces dernières années, ont recouru au terme anglais ethnolinguistics, avec ou sans épithète. Riley, Bartmiński et Underhill ont chacun élaboré une approche qui leur était propre; aucun des trois ne recourt à la MSN, encore que Bartmiński y fasse explicitement allusion. Ce qui relie ces travaux entre eux, et ce qui les relie aux nôtres, c'est un intérêt prononcé pour les rapports multiples et complexes entre langues, cultures et pensée, rapports que d'autres linguistes contemporains ne négligent pas pour autant, quoique l'importance qu'ils y attachent ne soit pas toujours la même et qu'il en reste pas mal qui n'y attachent aucune importance du tout ou bien qui en professent l'importance sans que cela ait le moindre impact sur leur façon de penser ${ }^{14}$.

41 Afin de différencier l'ethnolinguistique telle que nous la concevons dans nos propres écrits d'autres modèles ou approches qui portent le même nom, nous nous proposons de parler désormais, en français comme en anglais, d'ETHNOLINGUISTIQUE APPLIQUÉE, étiquette nettement moins usitée dans la littérature que le terme ethnolinguistique tout court. L'addition de l'adjectif appliqué est légitime, puisque l'ETHNOLINGUISTIQUe APPLIQUéE se destine avant tout aux étudiants de langues relativement avancés. Des diverses ethnolinguistiques évoquées dans ce qui précède, elle est la seule à être résolument pédagogique. Elle est en outre résolument abductive et recourt à la notion de saillance. Son objectif est double : il s'agit d'une part d'illustrer comment l'étude détaillée de mots, de métaphores, de tournures, de structures syntaxiques productives et de comportements communicatifs culturellement saillants peut conduire à la découverte de valeurs culturelles présumées, qui doivent à leur tour faire l'objet d'un examen approfondi lequel doit permettre de confirmer ou d'infirmer l'hypothèse initiale; et d'autre part, de voir comment, par le biais d'une étude détaillée de faits de société, de mots, de métaphores, de tournures, de structures syntaxiques productives et de comportements communicatifs culturellement saillants, il est possible de confirmer la réalité des valeurs culturelles que l'on a tendance à associer à des langues-cultures particulières.

\section{BIBLIOGRAPHIE}

Agar Michael, Language shock : understanding the culture of conversation, New York, William Morrow, 1994.

Agar Michael, The professional stranger, New York, Academic Press, 1996. 
André-Larochebouvy Danielle, La conversation quotidienne : introduction à l'analyse sémio-linguistique de la conversation, Paris, Didier-Crédif, 1984.

Bartmiński Jerzy, Aspects of cognitive ethnolinguistics, London, Equinox, 2009.

Béal Christine, « Les stratégies conversationnelles en français et en anglais : conventions ou reflet de divergences culturelles profondes ? ", Langue française, $n^{\circ}$ 98, 1993, pp. 79-106.

Béal Christine, Interactions quotidiennes en français et en anglais : de l'approche comparative à l'analyse des situations interculturelles, Bern, Peter Lang, 2010.

Bidet Alexandra, Quéré Louis, Truc Gérôme, « Ce à quoi nous tenons : Dewey et la formation des valeurs », in Dewey John, La formation des valeurs, Paris, La Découverte, 2011, pp. 5-64.

Blyth Carl, «The relevance of cultural linguistics to foreign language graduate education : from "language and culture" to "language as culture" ", in Allen Heather Willis, Maxim Hiram H. (eds), Educating the future foreign language professoriate for the $21^{\text {st }}$ century, Boston, Heinle, 2013, pp. 149-169.

Bréchon Pierre (ed.), Les valeurs des Français : évolutions de 1980 à 2000, Paris, Armand Colin, 2000.

Carroll Raymonde, Évidences invisibles : Américains et Français au quotidien, Paris, Seuil, 1987.

Chmelik Erzsébet, « Des énoncés para-doxaux aux observables de la sémantique », in Évrard Ivan, Pierrard Michel, Rosier Laurence, Van Raemdonck Dan (eds), Représentations du sens linguistique III , Bruxelles, De Boeck-Duculot, 2009, pp. 161-173.

Cohen-Émerique Margalit, «Le choc culturel, méthode de formation et outil de recherche », in Demorgon Jacques, Lipiansky Edmond-Marc (eds), Guide de l'interculturel en formation, Paris, Retz, 1999, pp. 301-315.

Dendale Patrick, Coltier Danielle, « Les adjectifs “psychologiques” et le marquage évidentiel de l'inférence », in Baider Fabienne, Cislaru Georgeta (eds), Cartographie des émotions : propositions linguistiques et sociolinguistiques, Paris, Presses Sorbonne Nouvelle, 2013, pp. 43-54.

Giora Rachel, On our mind : salience, context, and figurative language, Oxford : Oxford University Press, 2003.

Goddard Cliff, «Cultural values and "cultural scripts” of Malay (Bahasa Melayu) », Journal of pragmatics, $\mathrm{n}^{\circ} 27,1997$, pp. 183-201.

Goddard Cliff, « "Cultural scripts” and communicative style in Malay (Bahasa Melayu) », Anthropological linguistics, $n^{\circ}$ 42, 2000, pp. 81-106.

Goddard Cliff (ed.), Ethnopragmatics : understanding discourse in cultural context, Berlin, Mouton de Gruyter, 2006.

Goddard Cliff, Wierzbicka Anna, « Semantic primes and cultural scripts in language learning and intercultural communication », in Sharifian Farzad, Palmer Gary B. (eds), Applied cultural linguistics : implications for second language learning and intercultural communication, Amsterdam, John Benjamins, 2007, pp. 105-124.

González Hernández Ana Teresa, « De la langue à la culture : les stéréotypes comme vecteurs culturels dans l'enseignement/apprentissage du FLE », in Bermejo Larrea Esperanza, Corcuera Manso J. Fidel, Muela Ezquerra Julián (eds), Communications et écritures : autour de la linguistique et de la littérature françaises, Zaragoza, Prensas de la Universidad de Zaragoza, 2012, pp. 109-119.

Gremmo Marie-José, « Savoir apprendre, pouvoir apprendre sans se faire enseigner », Verbum, n • 18, 1995 / 1996, pp. 39-49. 
Gülich Elisabeth, «L'organisation conversationnelle des énoncés inachevés et de leur achèvement interactif en "situation de contact" ", DRLAV, nºs 34-35, 1986, pp. 161-182.

Hamon Philippe, 2008, « Qu'est-ce qu'une valeur? », in Prus Elena, GuŢu Ana (eds), La francopolyphonie : les valeurs de la francophonie, vol. 3, Chişinău, ULIM, 2008, pp. 9-13.

Inkova Olga (ed.), Saillance : aspects linguistiques et communicatifs de la mise en évidence dans un texte, vol. 1, Besançon, Presses universitaires de Franche-Comté, 2012.

d'Iribarne Philippe, L'étrangeté française, Paris, Seuil, 2006.

Jay Martin, Cultural semantics : keywords of our time, Amherst, University of Massachusetts Press, 1998.

Jeanneret Thérèse, La coénonciation en français : approches discursive, conversationnelle et syntaxique, Bern, Peter Lang, 1999.

Kohonen Susanna, « Turn-taking in conversation : overlaps and interruptions in intercultural talk », Cahiers de l'Association for French Language Studies, vol. XX, nº 1, 2004, pp. 15-32.

Levisen Carsten, Cultural semantics and social cognition : a case study on the Danish universe of meaning , Berlin, de Gruyter Mouton, 2012.

Loufrani Claude, « Locuteur collectif ou locuteur tout court », Recherches sur le français parlé, $\mathrm{n}^{\circ} 3$, 1981, pp. 215-243.

Loufrani Claude, « Le locuteur collectif : typologie de configurations discursives ", Recherches sur le français parlé, nº 6, 1985, pp. 169-193.

Matoré Georges, La méthode en lexicologie : domaine français, Paris, Didier, 1953.

Mejri Salah, Le figement lexical : descriptions linguistiques et structuration sémantique, Tunis, Publications de la Faculté des Lettres de la Manouba, 1997.

Palmer Gary B., Towards a theory of Cultural Linguistics, Texas, University of Texas Press, 1996.

Peeters Bert, " "S’engager” vs "to show restraint” : linguistic and cultural relativity in discourse management », in Niemeier Susanne, Dirven René (eds), Evidence for linguistic relativity, Amsterdam, John Benjamins, 2000, pp. 193-222.

Peeters Bert, « La quête de sens », Semiotica, n 145, 2003a, pp. 201-216.

Peeters Bert, «Le transculturel : sémantique, pragmatique et axiologie », La linguistique, vol. XXXIX, n 1, 2003b, pp. 119-133.

Peeters Bert, « Language and cultural values : the ethnolinguistic pathways model », Fulgor ( http://ehlt.flinders.edu.au/deptlang/fulgor/), vol. IV, n 1, 2009, pp. 59-73.

Peeters Bert, « La métalangue sémantique naturelle : acquis et défis », in François Jacques (ed.), Grandes voies et chemins de traverse de la sémantique cognitive, Leuven, Peeters, 2010a, pp. 75-101.

Peeters Bert, « "Un X peut en cacher un autre” : étude ethnosyntaxique », in Neveu Franck, Muni Toke Valelia, Klingler Thomas, Durand Jacques, Mondada Lorenza, Prévost Sophie (eds), CMLF $2010-2^{e}$ Congrès mondial de linguistique française, Paris, EDP Sciences, 2010b, pp. 1753-1775. http:// dx.doi.org/10.1051/cmlf/2010056.

Peeters Bert, «L'interculturel servi à la sauce MSN, ou À quoi sert la métalangue sémantique naturelle? ", in Auger Nathalie, Béal Christine, Demougin Françoise (eds), Interactions et interculturalité : variété des corpus et des approches, Bern, Peter Lang, 2012, pp. 149-180. 
Peeters Bert, « Language and cultural values : towards an applied ethnolinguistics for the foreign language classroom », in Peeters Bert, Mullan Kerry, Béal Christine (eds), Cross-culturally speaking, speaking cross-culturally, Cambridge, Cambridge Scholars Publishing, 2013a, pp. 231-259.

Peeters Bert, « Râler, râleur, râlite : discours, langue et valeurs culturelles », in Claudel Chantal, von Münchow Patricia, Pordeus Michele, Pugnière-Saavedra Frédéric, Tréguer-Felten Geneviève (eds), Cultures, discours, langues : nouveaux abordages, Limoges, Lambert-Lucas, 2013b, pp. 117-141.

Peeters Bert, «La langue de bois : un pèlerinage ethnolexicologique », in Marillaud Pierre, Gauthier Robert (eds), La mauvaise parole, $33^{\mathrm{e}}$ Colloque d'Albi Langages et Signification, Albi / Toulouse, CALS/CPST, 2013c, pp. 196-210.

Peeters Bert, « On va s'arranger / On s'arrangera : étude ethnophraséologique de deux actes (généralement) rassurants ", Scolia, n²8, 2014, pp. 129-149.

Peeters Bert, « La France de la débrouille : étude ethnoaxiologique d'une valeur culturelle hypothétique ", Revue de sémantique et pragmatique, à paraître a.

Peeters Bert, « Ah méfiance, quand tu tiens la France... », Cahiers de praxématique, à paraître b.

Peeters Bert, « Bienvenue au café du Commerce : propos ethnorhétoriques », Publif@rum, à paraître c.

Pottier Bernard, Sémantique générale, Paris, Presses Universitaires de France, 1992.

Py Bernard, « L'apprenant, le chercheur et les discours : quelques réflexions sur la notion de saillance », Tranel, $n^{\circ}$ 40, 2004, pp. 117-131.

Py Bernard, «Les données orales exolingues entre magma verbal, interlangue et langue ». Cahiers de l'ILSL, n²3, 2007, pp. 51-54.

Raccah Pierre-Yves, «Quelques remarques sur la sémantique linguistique et la construction du sens ", Travaux de linguistique et de philologie, n 30, 1992, pp. 69-81.

Raccah Pierre-Yves, « Lexique et idéologie : les points de vue qui s'expriment avant qu'on ait parlé », in Carel Marion (ed.), Les facettes du dire : hommage à Oswald Ducrot, Paris, Kimé, 2002, pp. 242-268.

Raccah Pierre-Yves, « Racines lexicales de l'argumentation : la cristallisation des points de vue dans les mots », Verbum, n³2, 2010, pp. 119-141.

Raccah Pierre-Yves, Várkonyi Zsófia, « “Assez”, c'est presque “trop” », in Neveu Franck, Muni Toke Valelia, Blumenthal Peter, Klingler Thomas, Ligas Pierluigi, Prévost Sophie, Teston-Bonnard Sandra (eds), $3^{e}$ Congrès Mondial de Linguistique Française, ILF / EDP Sciences, 2012, pp. 1909-1926. http://dx.doi.org/10.1051/shsconf/20120100146 [Version en ligne consultée le 16/01/2013]. Riley Philip, Language, culture and identity : an ethnolinguistic perspective, London, Continuum, 2007.

Sharifian Farzad, « Cultural Linguistics and intercultural communication », in Sharifian Farzad, Jamarani Maryam (eds), Language and intercultural communication in the new era, New York, Routledge, 2013, pp. 60-79.

Sharifian Farzad, "Advances in Cultural Linguistics », in Yamaguchi Masataka, Tay Dennis, Blount Benjamin (eds), Approaches to language, culture and cognition : the intersection of cognitive linguistics and linguistic anthropology, London, Palgrave McMillan, 2014, 99-123.

Sharifian Farzad, Palmer Gary B. (eds), Applied cultural linguistics : implications for second language learning and intercultural communication, Amsterdam, John Benjamins, 2007. 
Stoetzel Jean, Les valeurs du temps présent : une enquête européenne, Paris, Presses universitaires de France, 1983.

Underhill James W., Ethnolinguistics and cultural concepts : truth, love, hate and war, Cambridge, Cambridge University Press, 2012.

Vogel Klaus, Cormeraie Sylvette, «Du rôle de l'autonomie et de l'interculturalité dans l'étude des langues étrangères ", International Review of Applied Linguistics in Language Teaching, n 34, 1996, pp. 37-48.

Wierzbicka Anna, Understanding cultures through their key words : English, Russian, Polish, German, and Japanese, Oxford, Oxford University Press, 1997.

Wierzbicka Anna, Cross-cultural pragmatics : the semantics of human interaction, Berlin, Mouton de Gruyter, 2003 [ $\left.{ }^{1} 1991\right]$.

\section{NOTES}

1. Ce qui n'est pas facile : il convient de rappeler que, dans les mines d'or, on ne trouve pas non plus que de l'or...

2. Cette jolie formule est due à Tony Lattes (Université Montpellier 3), dont nous apprécions beaucoup la dextérité verbale.

3. Cf. Béal (2010: 14) : "On sait que les apprenants d'une langue seconde ont souvent des difficultés à communiquer (même s'ils ont d'assez bonnes compétences linguistiques) lorsqu'ils se retrouvent finalement à l'étranger pour un séjour prolongé, voire, de nos jours, pour une installation professionnelle. On parle alors de "choc culturel".»

4. Il aurait été possible de dire "On n'insistera jamais trop... », sans que cela change le sens de l'énoncé. Si, aux dires de Raccah et Várkonyi (2012), le comportement sémantique des adverbes assez et trop est assez proche - mais pas trop - ce n'est peut-être pas surprenant.

5. Les interactions rapportées par Kohonen (2004) proviennent d'enregistrements réalisés à table, dans un cercle familial.

6. Interview diffusée sur France 2, le 02/06/2006, dans le cadre de l'émission Les 4 vérités.

7. Les interactions rapportées par Béal $(1993,2010)$ proviennent d'enregistrements réalisés dans le siège melbournien d'une grande multinationale où des employés australiens et français se côtoient.

8. C'est nous qui soulignons.

9. Les points riches font évidemment écho aux faits surprenants de Peirce. Agar lui-même en convient dans plusieurs publications.

10. La plupart des auteurs situent celle-ci dans le prolongement des traditions philologiques, dialectologiques et anthropologiques de l'Allemagne du XIX ${ }^{\mathrm{e}}$ siècle, fortement influencées par la pensée de Wilhelm von Humboldt (1767-1835). Cet héritage allemand fut exporté aux États-Unis par Franz Boas (1858-1942), foundation professor of anthropology à l'Université de Columbia et père spirituel d'Edward Sapir (1884-1939), linguiste américain dont le nom restera à jamais associé, avec celui de son disciple Benjamin Lee Whorf (1897-1941), à la thèse de la relativité linguistique (la soi-disant hypothèse de Whorf et Sapir).

11. L'usage de PETITES MAJUSCULES sert de rappel au fait qu'on a affaire ici à des paradigmes très précis reliés à des prises de position théoriques particulières plutôt qu'à des domaines plus vastes. Il y a ainsi une différence entre la LINGUISTIQUE COGNITIVE, dont les chefs de file sont George Lakoff et Ronald W. Langacker, et la linguistique cognitive, dénomination qui convient à toute approche de la linguistique qui se préoccupe de la cognition (y inclus les différents courants existant au sein de la linguistique générative). En anglais et dans certaines autres langues, on 
recourt souvent à des majuscules initiales pour opposer le paradigme précis (Cognitive Linguistics) au cadre plus vaste (cognitive linguistics).

12. Pour une présentation rapide, en français, de l'approche MSN, on verra Peeters (2010a, 2012). 13. Le terme mot clé s'utilise depuis longtemps pour renvoyer à des mots de fréquence élevée (le plus souvent des noms communs, des adjectifs ou des verbes), parfois difficiles à traduire, culturellement plus «chargés" que d'autres, des mots qui sont "particularly important and revealing in a given culture » (Wierzbicka $1997: 15-16$ ), qui assument pour ainsi dire « more than their share of cultural work" (Jay $1998: 4$ ), dont le sens est "pivotal for social interaction and societal life» (Levisen 2012 : ix). Au sein de la linguistique et en particulier de la lexicologie françaises, ce sont les travaux de Georges Matoré, à commencer par Matoré (1953), qui ont assuré le succès du terme.

14. Un grand nombre de linguistes qui s'intéressent explicitement aux rapports entre langues, cultures et pensée travaillent dans le domaine de la linguistique appliquée à l'enseignement des langues. On y ajoutera ceux qui se réclament de la LINGUISTIQUe CULTURELLE (Cultural Linguistics) de Palmer (1996), dont les incursions en linguistique appliquée ont donné lieu à un volume (Sharifian, Palmer 2007) auquel ont notamment participé Anna Wierzbicka et Cliff Goddard (Goddard, Wierzbicka 2007). Pour une présentation toute récente de la LINGUISTIQUE CULTURELLE, on verra Sharifian $(2013,2014)$; pour un exemple des répercussions qu'elle peut avoir dans le cadre de la formation en FLE, voir Blyth (2013).

\section{RÉSUMÉS}

Dans une interaction qui met en présence des interlocuteurs n'appartenant pas à la même langue-culture, les éventuels malentendus ou échecs communicatifs qui surgissent peuvent être occasionnés par des différences culturelles ignorées. Dans une situation de ce genre, surtout si elle est susceptible de se répéter, il sera utile que les interlocuteurs se familiarisent avec les valeurs culturelles les plus importantes dans la langue-culture de l'autre - ou qu'au moins l'un d'eux fasse un effort allant dans ce sens. Mais comment s'y prendra-t-on? Comment se familiariser avec quelque chose d'aussi intangible qu'un ensemble de valeurs? Dans cet article, nous proposons un certain nombre de stratégies d'apprentissage de valeurs "étrangères » dans le cadre d'une formation en langues étrangères; l'exploitation intelligente de certaines ressources de la langue enseignée, l'analyse sélective de certains comportements communicatifs et l'observation de certains faits de société judicieusement choisis donnent à l'enseignant le moyen d'équiper ses étudiants avec des savoirs culturels pertinents. Nous appelons ETHNOLINGUISTIQUE APPLIQUÉE l'ensemble des démarches permettant à des étudiants de niveau relativement avancé de voir plus clair dans la problématique des langues-cultures et des valeurs culturelles qu'on a coutume d'y associer.

The potential misunderstandings or communicative failures that occasionally arise in interactions between speakers whose primary languaculture is different may be due to cultural differences that go unnoticed. In this kind of situations, even more so when they are bound to recur, it will pay off for the speakers to familiarise themselves with the main cultural values in their speech partner's languaculture - or at least for one of them to make an effort going in that direction. But this is easier said than done. How does one become familiar with something as intangible as a set of values? In this paper, we suggest a number of learning strategies for 
"foreign" values in a foreign classroom setting; the intelligent exploitation of selected resources that are part of the language taught, the analysis of certain communicative behaviours and the observation of carefully chosen salient features of a society provide language teachers with the means to equip their students with relevant cultural knowledge. APPLIED ETHNOLINGUISTICS is the set of pathways allowing students who have reached a relatively advanced competency level in their chosen foreign language to gain a clearer understanding of the language-culture nexus and of the cultural values that tend to be associated with particular languacultures.

INDEX

Mots-clés : Valeurs culturelles ; langues-cultures étrangères ; ethnolinguistique appliquée ; stratégies d'apprentissage ; communication interculturelle

Keywords : Cultural values; foreign languacultures; applied ethnolinguistics; learning strategies; intercultural communication

\section{AUTEUR}

\section{BERT PEETERS}

Brisbane, Australie 